\title{
Physiological impacts of ABA-JA interactions under water-limitation
}

\author{
Carlos de Ollas $^{1} \cdot$ Ian C. Dodd ${ }^{1}$
}

Received: 18 September 2015/Accepted: 18 May 2016/Published online: 14 June 2016

(C) The Author(s) 2016. This article is published with open access at Springerlink.com

\begin{abstract}
Plant responses to drought stress depend on highly regulated signal transduction pathways with multiple interactions. This complex crosstalk can lead to a physiological outcome of drought avoidance or tolerance/resistance. ABA is the principal mediator of these responses due to the regulation of stomatal closure that determines plant growth and survival, but also other strategies of drought resistance such as osmotic adjustment. However, other hormones such as JA seem responsible for regulating a subset of plant responses to drought by regulating $\mathrm{ABA}$ biosynthesis and accumulation and ABA-dependent signalling, but also by ABA independent pathways. Here, we review recent reports of ABA-JA hormonal and molecular interactions within a physiological framework of drought tolerance. Understanding the physiological significance of this complex regulation offers opportunities to find strategies of drought tolerance that avoid unwanted side effects that limit growth and yield, and may allow biotechnological crop improvement.
\end{abstract}

Keywords ABA - JA - Drought tolerance - Crosstalk . Signalling $\cdot$ Stomatal closure

\section{Introduction}

At both tissue and whole plant levels, abscisic acid (ABA) is the most important hormone controlling plant water loss and hence plant water status and performance in water limited

Carlos de Ollas

deollas@uji.es

Ian C. Dodd

i.dodd@lancaster.ac.uk

1 Lancaster Environment Centre, Lancaster University, Lancaster, UK conditions. ABA is the main driver of stomatal closure which is probably the most effective mechanism to minimise dehydration. In terms of water stress sensing, stomata and roots are the most important organs that integrate environmental conditions with plant water status, with an important distinction depending on the origin (root or leaf) of the stress sensation (Kramer 1988; Passioura 1996). Both soil moisture and vapour pressure deficit (VPD) can influence plant responses to water stress, even if these environmental conditions have a similar outcome (e.g. stomatal closure). In response to drought, plants can exhibit a strategy of drought escape/avoidance or drought tolerance. Avoidance implies the maintenance of tissue water potential even in conditions of low soil moisture whereas tolerance indicates the ability to withstand water deficits that lower tissue water potential. Mechanisms of drought tolerance imply the biosynthesis of functional proteins in response to drought like molecular chaperones, hydrophilic proteins [dehydrins and late embryogenesis abundant (LEA) proteins] and enzymes involved in the synthesis of osmoprotectants (proline and sugars). Roots "sense" soil drying thereby upregulating ABA synthesis, some of which may be transported in the xylem to the shoots (Wilkinson and Davies 2002; Puértolas et al. 2013). Leaves must integrate multiple environmental signals including these chemical/hydraulic signals transported via the xylem sap, as well as light, $\mathrm{CO}_{2}$ concentration, VPD and temperature.

At the molecular level, there are thousands of ABA dependent genes controlling growth, senescence, secondary metabolism, protein biosynthesis and osmotic adjustment to coordinate plant behaviour under stress conditions (Yoshida et al. 2014). Classically, these studies of drought related gene expression have been divided into ABA-dependent and $\mathrm{ABA}$-independent branches of water stress signalling (extensively reviewed by Yamaguchi-Shinozaki 
and Shinozaki 2006; Roychoudhury et al. 2013; Yoshida et al. 2014). A mosaic of transcription factors (TFs) and genes control several pathways and modify expression of the corresponding ABA-responsive or water stress-responsive genes. Nevertheless a more subtle network of water stress signalling has emerged in the last decade, with other hormones like jasmonic acid (JA), ethylene, auxin, cytokinin and brassinosteroids playing diverse roles to fine tune ABA biosynthesis and hence water stress signalling/plant physiological responses. This can be defined as hormonal and molecular crosstalk.

Most of the molecular knowledge of plant responses to water deficit comes from laboratory experiments where plants experience dehydration due to water withdrawal (Harb et al. 2010; Cheng et al. 2013) or osmotic treatments (Weng et al. 2011; Liu et al. 2012). While these experiments unveiled genetic networks of stress signalling, plant genotypes can be wrongly classified as stress tolerant if they survive by avoiding dehydration (by limiting water losses). Measuring plant water status (tissue water content/water and osmotic potential/turgor) is necessary to determine if plants show drought tolerance (improved performance at the same water status). Understanding both hormonal crosstalk and physiological responses are necessary if agronomists are to exploit drought avoidance/tolerance mechanisms in farmers' fields. Lately, JA has gained much attention as it participates in abiotic stress responses [recently reviewed by Kazan (2015)] in addition to its involvement in biotic stress (Wasternack 2014). Jasmonic acid is an attractive topic of research due to its participation in multiple stress signalling responses. For example, in biotic stress it is well known that JA plays an antagonistic role to salicylic acid but works synergistically with Ethylene (ET) (Hirayama and Shinozaki 2010). In contrast, the relation of JA with other hormones (mainly ABA and ET) in response to abiotic stress is still being studied. The fact that JA presents such a multifaceted behaviour and is implicated in a broad range of common stress elicitors are powerful reasons to draw our attention, especially if manipulating these interactions offers the possibility to improve crop tolerance to both biotic and abiotic conditions. By focusing on ABA-JA interactions, this article aims to summarize the participation of JA in plant physiological responses and its molecular regulation to drought leading to tolerance/adaptation. We briefly summarize the molecular interactions of ABA and JA signalling leading to meaningful physiological responses to drought.

\section{Tissue ABA/JA accumulation}

ABA accumulates in shoots (Zhang et al. 2012) and roots (Puértolas et al. 2013) even under optimal conditions, but this accumulation is stimulated by any decrease in cellular turgor. The initial mechanism linking the stress sensation and $\mathrm{ABA}$ biosynthesis is still unknown, but ABA biosynthesis under stress conditions is directly correlated with plant/tissue water status. Although ABA has many biosynthetic steps [as described in Nambara and MarionPoll (2005)], NCED seems to be the key biosynthetic enzyme in the pathway (Thompson et al. 2007) because its transcription is well correlated with plant water status and mutants that overexpress NCED, and lines transformed with multiple copies of NCED accumulate more ABA (Thompson et al. 2000). Nevertheless, local ABA concentration is a balance between biosynthesis, catabolism and transport between cells and tissues (e.g. root-to-shoot) (Nambara and Marion-Poll 2005; Jiang and Hartung 2008; Munemasa et al. 2015).

Jasmonic acid is synthesized via the octadecanoid pathway under multiple (both biotic and abiotic) stress conditions, and is associated with resistance to biotic stress and wounding in stress signalling. Of the JA precursors, 12-oxophytodienic acid (OPDA) is one of the most important due to its possible independent roles in signalling downstream of JA. The Isoleucine conjugate form of JA, jasmonic acid Isoleucine (JA-Ile) has been characterized as the key signalling compound in JA-dependent responses due to its interaction with the receptor Coronatine-Insensitive 1 (COI1). Several recent publications have demonstrated JA's involvement in water stress responses (Brossa et al. 2011; De Ollas et al. 2015). JA and ABA seem to share common targets and crosstalk between both signalling pathways is extensive. JA application $(5-15 \mu \mathrm{M}$ in hydroponic solution) enhanced foliar ABA concentration 4-7-fold (Bandurska et al. 2003) and JA deficiency (jar1) can diminish ABA accumulation (De Ollas et al. 2013). It is important to point out that ABA concentration usually increases as tissue water status declines (Puértolas et al. 2013), while JA concentration fluctuates and seems to accumulate during early stages of stress. While JA accumulation in response to dehydration has been suggested (Arbona et al. 2010), its involvement in molecular and physiological responses, either in concert with ABA or acting independently, is still unclear. Importantly, after transient JA accumulation, JA-dependent signalling may remain active (Wasternack 2014).

\section{Molecular responses to $\mathbf{A B A} / \mathbf{J A}$}

ABA-dependent signalling has several branches controlled by ABA-responsive elements (ABREs), NAC and MYC/ MYB TFs (Fig. 1-Signal transduction). The ABREbranch of gene expression requires multiple ABREs, or the combination of an ABRE with a coupling element (Zhang et al. 2005). Usually a single ABRE is insufficient for the 


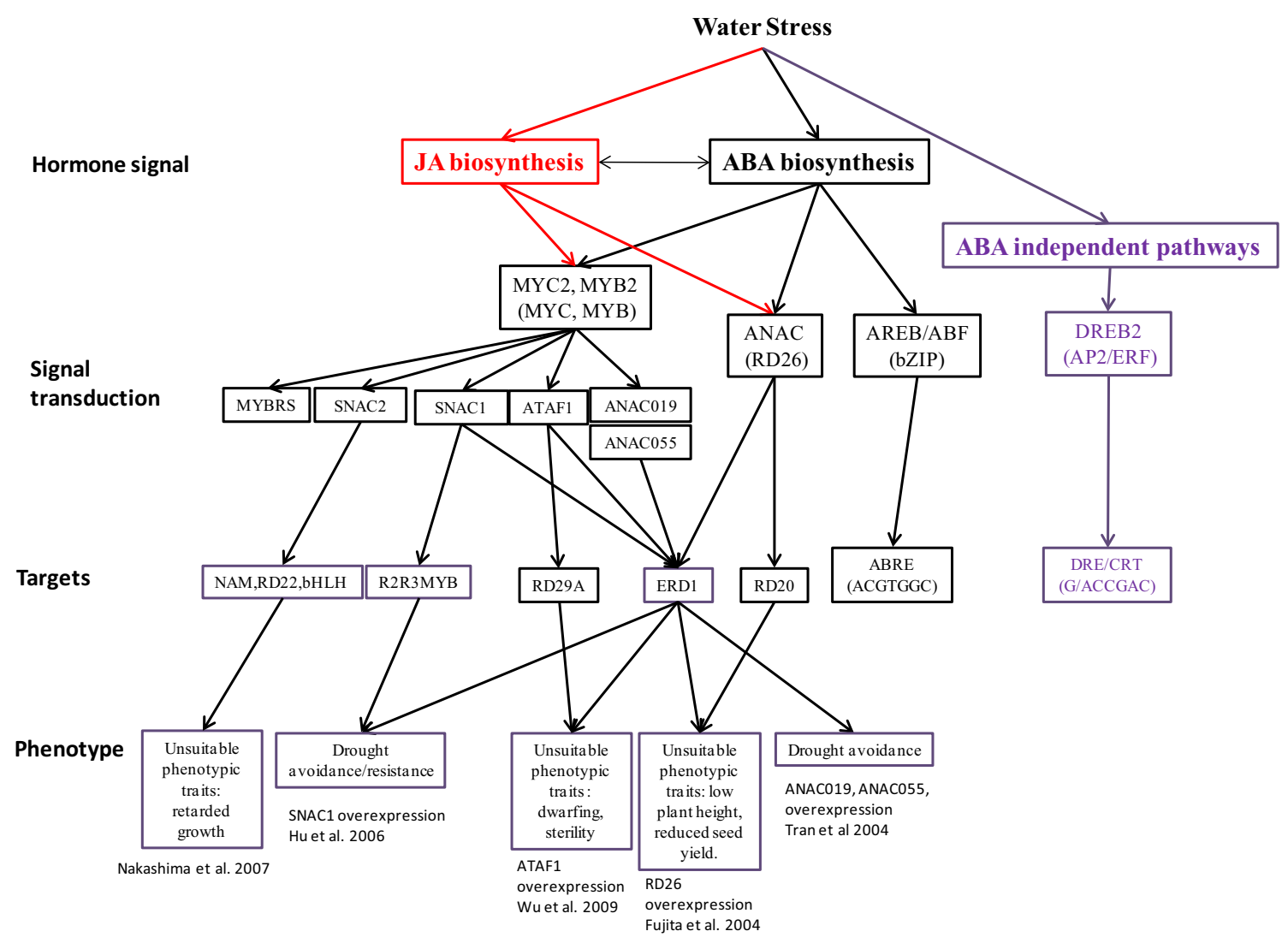

Fig. 1 Summary of signalling pathways related to JA-ABA interaction in response to drought. Briefly, water deficit can enhance both $\mathrm{ABA}$ and JA accumulation. Water stress also activates ABA independent pathways via DREB2 transcription factors targeting genes with DRE sequences in their promoters. The ABA dependent pathway uses AREB/ABF, ANAC and MYC transcription factors.

ABA dependent initiation of transcription; successful initiation requires another ABRE element or another type of coupling element like CE1 (coupling element 1), CE3, motif III, or a drought-responsive element (DRE)/CRT (Umezawa et al. 2010). Among the group AbZIP subfamily, AREB1/ABF2, AREB2/ABF4 and ABF3 are master TFs that cooperatively regulate ABRE-dependent gene expression in ABA signalling under stress conditions. In addition to AREB/ABF, there are other TFs involved in ABA-mediated gene expression in response to abiotic stress. The AP2/ERF proteins play a central role in stress signalling (Liu et al. 2000), while the subfamily of DRE/ CRT binding proteins (DREB1/CBFs) can bind to the DRE cis-acting element. DREB1D/CBF4 and the cold-responsive DREB1A-C/CBF1-3 are also induced by exogenous ABA. On the other hand, DREB2A and DREB2B are strongly induced by osmotic stress but only slightly by ABA (Umezawa et al. 2010). This requirement for multiple heterogeneous or homogeneous elements of regulation offers the possibility of controlling transcription by tuning
AREB seems to be ABA-specific targeting ABRE sequences, ANAC and MYC seem to be activated by JA and ABA. Overexpression of each of these transcription factors activates a subset of genes giving a specific phenotype of "drought tolerance" indicated at the base of the figure

the ABA concentration in a tissue (two ABRES) or initiating transcription by the action of ABA and another signalling input (ABRE and a coupling element). This system is more plastic and better able to adapt to specific environmental conditions.

On the other hand, JA dependent signalling is initiated by the interaction of JA-Ile with the receptor COI1 which releases a series of molecular responses that involve interactions with many signalling components including the JAZ proteins and MYC2 TF's (reviewed by Wasternack 2014). ABA and JA signalling pathways can interact at several points, suggesting a role for JA in the response to water stress. MYC2 and MYB2 TF's are involved in ABAmediated gene expression in Arabidopsis (Kazan and Manners 2013). Transgenic plants overexpressing MYB2 and MYC2 show enhanced sensitivity to ABA and have lower electrolyte leakage following cessation of watering (Abe et al. 1997, 2003), likely because enhanced stomatal closure limits dehydration. The induction of MYC2 by ABA seems to rely on the JA receptor COI1 (Lorenzo et al. 
2004). In Oryza sativa, the MYC-family TF OsbHLH148 interacted with OsJAZs in response to drought (Seo et al. 2011). A model for the OsbHLH148-related jasmonate signalling pathway in drought stress was proposed such that $\mathrm{ABA}$ and JA act synergistically in response to stress with JA acting upstream of ABA.

Overexpressing OsbHLH148 plants reportedly had a drought tolerant phenotype associated with the expression of OsDREB1. Transformed rice plants had a higher survival rate (77-92\%) than WT plants $(9 \%)$ when water was withheld, and detached air-dried leaves had a higher photosystem II efficiency (Fv/Fm) than non-transformed plants. This suggests greater stomatal closure to maintain leaf water status and protect the photosystems. It is uncertain whether these specific mechanisms will be relevant to maintaining yields in dryland rice production. In contrast, another publication dealing with MYC2-related signalling reported that after ceasing watering, then maintaining a moderate drought by daily irrigation (leaf relative water content (RWC) averaged $65 \%$ ), both coi-1 (insensitive to JA) and jin-1 (insensitive to the MYC2 dependent branch of JA signalling) mutants showed no biomass reduction while this treatment approximately halved biomass of WT and jar1 plants (Harb et al. 2010). This response was unrelated to the biomass (and presumably transpiration rate) of coi-1 plants, which grew similarly to WT plants under well-watered conditions. Thus perturbing JA signalling may enhance biomass accumulation, but further work is required to evaluate the generality of this response, since any trait may confer drought tolerance under specific drought conditions (Tardieu 2012).

The Ethylene (ET) transcription factor family are interesting targets for both JA-ABA interactions and ET. ERF5 and ERF6 are two redundant TF that are involved in leaf growth inhibition under mild drought stress (Dubois et al. 2013), with the expression of these genes being JA/ ET dependent (Moffat et al. 2012). In contrast, ERF11 (responsive to ABA and JA) acts antagonistically to ERF6, with ABA inactivating ERF6 under water deficit (Sewelam et al. 2013). Another member of ERF family, ERF1, can be activated by ET, JA or by the synergistic combination of both hormones (Lorenzo et al. 2003) and seems a point of convergence of both hormonal pathways. Although ERF1 overexpression reportedly enhanced drought tolerance, no details of the drought selection were explained (Weiste et al. 2007). When 35S:ERF1 plants displayed resistance to various stresses (high salinity, drought and mannitol), ERF1 expression increased rapidly (within 1-2 $\mathrm{h}$ of stress imposition) and transiently (Cheng et al. 2013), similar to the pattern of JA accumulation in response to dehydration (De Ollas et al. 2013). Interestingly, foliar spraying with $50 \mu \mathrm{M}$ ABA decreased ERF1 expression, thereby overriding JA and ET induction. Since the drought resistance phenotype was based on plant survival rate following water withdrawal and recovery (90\% for overexpressing lines versus $33 \%$ for WT), overexpressing lines avoided drought by reduced transpiration rate due to enhanced stomatal closure. Moreover, stomata of 35S:ERF1 plants were more closed even in well-watered conditions, probably implying a lower growth rate in optimal conditions. Also, these plants seem to accumulate more proline and ABA in well-watered conditions, an undesirable trait in any crop according to a comprehensive analysis of various conceptual ABA ideotypes (Blum 2015). ERF has 122 members in Arabidopsis, and the complexity of the interaction of this network with ABA-JA-ET and other players in abiotic conditions makes it difficult to create useful models (synergism versus antagonism of ABA-JA in water stress and interaction with ET) requiring caution in assessing phenotypes.

Several NAC TF's are upregulated by ABA, salinity and dehydration and it is possible that ANAC055 and RD26/ ANAC072 proteins bind to the CATGTG motif and regulate stress inducible genes associated with drought tolerance. ANAC019 and ANAC055 are active in E3 ligase modulation of ABA signalling, playing dual roles in regulation of ABA and JA responses (Fujita et al. 2011). Since both of these TF's are involved in both JA and ABA signalling (Fig. 1), ABA- and JA-insensitive mutants are necessary to discriminate the pathways involved in drought tolerance. ABA-induced expression of ANAC019 and ANAC055 is impaired in coil-1 and jail/jinl (Jiang et al. 2009), reinforcing the participation of those TFs in crosstalk between JA and ABA pathways. Moreover, an involvement of ANAC019 and ANAC055 in drought stress regulation was reported because they bind specifically to the promoter region of ERD1, a dehydration responsive element. More recently, constitutive overexpression of a Miscanthus lutarioriparius NAC gene (MINAC5) enhanced drought and cold tolerance in Arabidopsis by inducing ABA hypersensitivity (Yang et al. 2015), as in overexpression of other TF's such as OsbHLH148. If these TF's are able to improve mechanisms allowing higher photosynthesis even when stomata are more closed (higher water use efficiency) due to protective mechanisms under low soil moisture, they may improve drought tolerance.

Several examples of genuine drought tolerance associated with the NAC family of TF exist, and the effects of NAC overexpression under stress conditions has been reviewed (Puranik et al. 2012). SNAC-1 (STRESS RESPONSIVE NAC 1) is upregulated upon treatment with both ABA and JA (Nakashima et al. 2012). Overexpressing SNAC-1 in rice plants delayed leaf rolling and reduced water loss (drought avoidance) in field-grown plants at anthesis (Hu et al. 2006). Interestingly, despite having up to $20 \%$ lower stomatal conductance than WT plants, 
photosynthetic rate was similar to WT plants, and these overexpressing plants re-established turgor pressure at a lower RWC upon re-watering, suggesting enhanced drought tolerance was conferred by improved osmotic adjustment. On the other hand, constitutive overexpression of OsNAC6 (SNAC-2) in rice retarded growth and decreased productivity, although survival rate after desiccation was higher (Nakashima et al. 2007). OsNAC6/ SNAC-2 is induced by cold, salt drought, ABA and JA (Ohnishi et al. 2005). However, root specific (RCc3 promoter) overexpression of the NAC family OsNAC10 in Oryza sativa significantly enhanced drought tolerance at the reproductive stage, increasing grain yield by $25-42 \%$ and by $5-14 \%$ over controls in the field (with more filled grains and spikelets) under drought (rain was excluded from 10 days before heading to 20 days after heading) and normal conditions respectively (Jeong et al. 2010). This may be related to a more extensive root system, thereby maintaining water uptake. In contrast, constitutive (GOS2) overexpression of OsNAC10 throughout the plant (including the floral organs) greatly decreased grain filling rate under both normal and drought conditions. Since improved root development is a common strategy for drought avoidance; using root specific promoters (Ghanem et al. 2011) may represent a practical strategy to harness beneficial NAC-dependent traits without some of the deleterious side-effects resulting from constitutive overexpression.

Due to space limitations we have not addressed the involvement of WRKY TF family involvement in this topic, partly because of the complexity of the family and the multiple interactions. Nevertheless recent work illustrates the involvement of WRKY TF family in ABA-JA response to drought stress (Rabara et al. 2015).

\section{Physiological responses to ABA/JA}

Plants cope with water stress by closing their stomata to avoid water loss. Increased ABA concentrations induce stomatal closure via intracellular signalling in the guard cells. Cytosolic production of nitric oxide (NO) and reactive oxygen species (ROS) (Fig. 2), due to the interaction of ABA with PP2C (ABA receptor) and SnRK2 (ABA dependent phosphorylase), causes fluctuations in symplastic $\mathrm{Ca}^{2+}$ concentrations (induced by the activity of several ion transporters). This causes ion efflux thereby lowering cellular turgor due to water efflux out of the guard cells and hence causing stomatal closure. This signal transduction pathway, along with involvement of JA and other hormones was recently reviewed (Daszkowska-Golec and Szarejko 2013). In addition to the classical direct involvement of ABA-mediated stomatal closure via $\mathrm{ABA}$ signalling in the guard cells, it can also indirectly induce stomatal closure by decreasing leaf hydraulic conductance (Pantin et al. 2013). While the ABA insensitive mutants abil-1 and abi2-1 displayed stomatal closure when detached leaves of Arabidopsis were fed $50 \mu \mathrm{M}$ ABA via the transpiration stream, the slac $1-1$ and ost $2-2$ mutants (Fig. 2) were insensitive to ABA (Pantin et al. 2013). To explain this disparity and the fact that plants defective in ABA signalling closed their stomata under high VPD, ABA inactivation of bundle sheath aquaporins (PIPs) was postulated based on (Shatil-Cohen et al. 2011). Briefly, an ABA dependent signalling pathway decreases PIP aquaporin activity thus reducing leaf hydraulic conductance and decreasing leaf apoplast water potential (Fig. 2), although whether ABA inhibits or stimulates hydraulic conductance is concentration-dependent (Dodd 2013). This work suggests an unexplained ABA signalling that is independent of ABI1-2, ABI2, SLAC and OST in decreasing leaf conductance, and that ABA may modify other hormonal pathways thereby affecting PIP activity. Due to the extensive hormonal crosstalk that can influence stomatal closure, excluding the influence of other hormones would further establish this interesting model of chemical/hydraulic regulation.

It is well established that ABA plays a central role in regulating stomatal closure (Daszkowska-Golec and Szarejko 2013; Dodd 2013) yet other phytohormones can also have direct effects and/or mediate the influence of ABA. JA's involvement in stomatal closure is not completely understood, even though many experiments have exposed isolated epidermis to various agents (Table 1). Exogenous application of either ABA or MeJA to detached leaves elicited stomatal closure (Suhita et al. 2004). In contrast, it was suggested that MeJA needs a pool of ABA to be effective in closing stomata, since MeJA treatment was ineffective in closing stomata in isolated epidermis of ABA deficient mutants $(a b a)$ and plants treated with the ABA biosynthesis inhibitor fluridone (Hossain et al. 2011). Thus MeJA may exercise its action by increasing ABA biosynthesis, as previously suggested (Kim et al. 2009) in MeJA overaccumulating rice. Alternatively, MeJA treatment did not elicit stomatal closure in ABA deficient mutants but ABA treatment did; suggesting that ABA acts downstream of coil, while in abi1-2 neither ABA nor MeJA elicited stomatal closure. The authors suggested a model of independent MeJA and ABA signalling converging upstream of the second messengers (ROS and NO) induction (Munemasa et al. 2011). A schematic of these interactions is summarised in Fig. 2.

However, there are also some discrepancies in the direct effect of exogenous treatment of MeJA on stomatal closure (Table 1). In Arabidopsis (Col-0), MeJA only reduces stomatal aperture at concentrations exceeding $200 \mu \mathrm{M}$ 


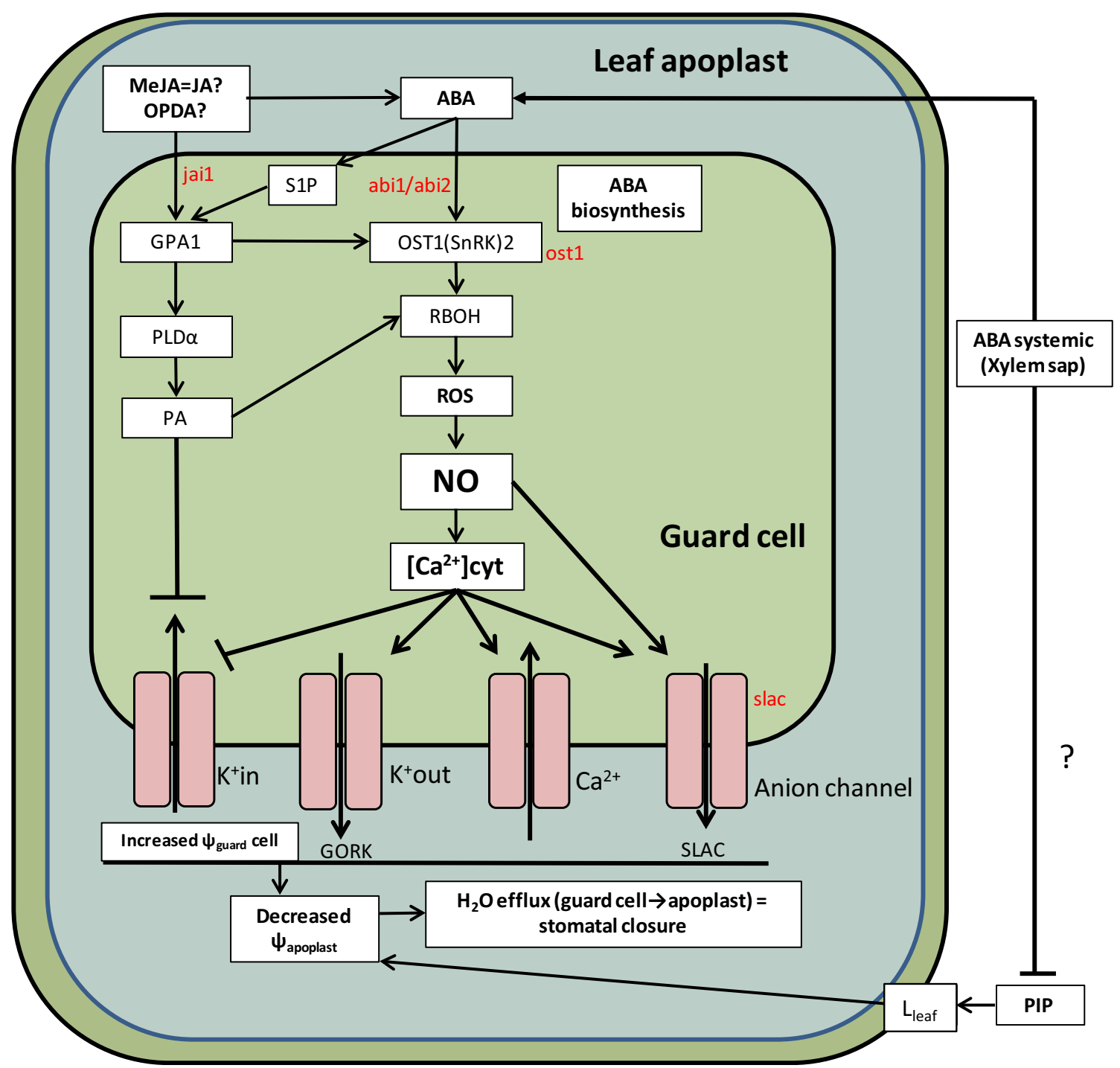

Fig. 2 Stomatal closure signalling pathways. ABA produced in the guard cells or imported to the apoplast from other tissues unleashes the production of $\mathrm{NO}$ and $\mathrm{ROS}$, the subsequent release of $\mathrm{Ca}^{2+}$ controls the activity of several ion channels causing decreased osmotic pressure in the cell, leading to $\mathrm{H}_{2} \mathrm{O}$ efflux and stomatal closure. This pathway can be activated by MeJA. Question marks highlight the lack of information on the individual activity of JA, MeJA and OPDA activating this pathway or modifying ABA

(Savchenko et al. 2014) whereas almost complete stomatal closure occurred in epidermal peels of Arabidopsis (Ler and Col-0) treated with either MeJA or ABA at much lower concentrations (10-20 $\mu \mathrm{M})$ (Suhita et al. 2004). In contrast to these results and also in epidermal peels of Arabidopsis, concentrations up to $100 \mu \mathrm{M}$ MeJA had no effect on stomatal aperture but lower concentrations of OPDA (precursor of JA) decreased stomatal aperture (Montillet et al. 2013). According to Savchenko's model, OPDA promotes stomatal closure independently from JA and in cooperation with ABA. Nevertheless, treatment with biosynthesis. An alternative hydraulic pathway activated by ABA (Pantin et al. 2013) is described where ABA deactivates PIP aquaporins in the bundle sheath decreasing leaf hydraulic conductivity $\left(\mathrm{L}_{\text {leaf }}\right)$, which will decrease $\psi_{\text {leaf }}$ and $\mathrm{g}_{\mathrm{s}}$. Question marks reflect steps that are theorized but not sustained by experimental data. Mutants in red designate an interruption in the pathway that has been experimentally verified

1-10 $\mu \mathrm{M}$ of ABA or MeJA (Suhita et al. 2004) was much less effective in closing stomata of the jar1-1 mutant (defective in conjugating isoleucine to JA), suggesting that MeJA treatment is JA-Ile dependent and hence COI-1 dependent. These experiments expose the variability of the stomatal responses to exogenous hormonal treatment and the necessity of assessing apoplastic hormone concentration adjacent to the guard cells to better compare epidermal bioassays with in planta experiments. This is important to distinguish hormonal crosstalk (JA influencing ABA biosynthesis in this particular example) from molecular 
Table 1 Summary of stomatal closure following ABA, MeJA and OPDA exogenous treatments to epidermal peels of Col-0 or Landsberg erecta

\begin{tabular}{lclll}
\hline $\begin{array}{l}\text { Hormonal } \\
\text { treatment }\end{array}$ & $\begin{array}{l}\text { Concentration } \\
(\mathrm{uM})\end{array}$ & $\begin{array}{l}\text { Stomatal } \\
\text { closure }(\%)\end{array}$ & Genotype & Source \\
\hline ABA & 1 & 50 & Col-0 & Savchenko et al. (2014) \\
ABA & 10 & 80 & Col-0 & Montillet et al. (2013) \\
ABA & 1 & 30 & Col-0 & Munemasa et al. (2007) \\
ABA & 10 & 25 & Col-0 & Hossain et al. (2011) \\
ABA and MeJA & 1 & 50 & Col-0 & Montillet et al. (2013) \\
ABA or MeJA & 10 & 70 & Ler & Suhita et al. (2004) \\
MeJA & 50 & 50 & Col-0 & Savchenko et al. (2014) \\
MeJA & 1 & n.s. & Col-0 & Montillet et al. (2013) \\
MeJA & 1 & 25 & Col-0 & Munemasa et al. (2007) \\
MeJA & 10 & 25 & Col-0 & Hossain et al. (2011) \\
12-OPDA & 10 & 50 & Col-0 & Savchenko et al. (2014) \\
\hline
\end{tabular}

$n s$ not significant interactions in a signalling pathway leading to a physiological outcome (degree of stomatal closure).

Although water uptake is controlled by stomatal opening, changes in root hydraulic conductivity $\left(\mathrm{L}_{\mathrm{pr}}\right)$ can also influence plant water status to mediate transpiration. While it is important not to underestimate the importance of root morphology and anatomy in mediating $\mathrm{L}_{\mathrm{pr}}$ (Vadez 2014), regulation of cell-to-cell water transport might also play an important role in maintaining water status under moderate water stress. Nevertheless, soil water conductivity is likely more limiting under severe water deficits (Draye et al. 2010). Several publications highlighted the involvement of exogenous ABA application $(100 \mu \mathrm{M})$ (Aroca et al. 2008; Kudoyarova et al. 2011) and transgenic plants with enhanced root ABA concentrations (Thompson et al. 2007) in enhancing $\mathrm{L}_{\mathrm{pr}}$. Nevertheless, applying higher $\mathrm{ABA}$ concentrations to the root system can decrease $\mathrm{L}_{\mathrm{pr}}$ (reviewed in Dodd 2013). Hormonal regulation of $\mathrm{L}_{\mathrm{pr}}$ is an attractive topic for further research, but since plant water status seems primarily regulated by stomatal responses, it is uncertain whether changing $\mathrm{L}_{\mathrm{pr}}$ can improve plant water status thereby promoting drought tolerance.

Supplying MeJA $(10-100 \mu \mathrm{M})$ in nutrient solution can also increase $\mathrm{L}_{\text {pr }}$ of WT tomato plants, while plants deficient in JA (def-1) had a lower $\mathrm{L}_{\mathrm{pr}}$ (Sánchez-Romera et al. 2014). Exogenous application of $100 \mu \mathrm{M}$ MeJA rescued this phenotype in JA deficient mutants. Although the short term response $(1 \mathrm{~h})$ was partially dependent on $\mathrm{ABA}$ (treatment with the ABA biosynthesis inhibitor fluridone partially abolished the MeJA effect), the long term effect was independent of ABA. MeJA treatment modified the expression and activity of PIP aquaporins, with the expression of some isoforms downregulated and others upregulated. Such tissue specific regulation of aquaporins by hormones under stress conditions can link hormonal dependent signalling with hydraulic regulation.

\section{Conclusions and future scope}

While jasmonates seem involved in drought stress responses, a more comprehensive assessment of environmental and physiological responses (soil and plant water status, stomatal closure and photosynthesis) are necessary to understand the molecular mechanisms that can potentially improve crop yields in water-limited environments. Assessing $\mathrm{ABA}$ and JA interactions in drought stress responses needs to consider the three levels of interactions summarised in this short review (biosynthetic, molecular and physiological). Accurate hormonal quantification of ABA and JA, JA-Ile and ideally OPDA in relevant tissues is necessary (Dave et al. 2011) to assess the real players in most physiological responses, since both hormonal pathways can regulate the other, and to identify the bioactive molecule(s) in these interactions (OPDA versus JA and JAIle). We also need to assess the direct influence of jasmonates in responses to drought as distinct from the indirect influence through $\mathrm{ABA}$ biosynthesis and signalling. It is important to unveil these interactions because they offer an opportunity to exploit the natural complexity of drought signalling. This may allow us to enhance crop performance by inducing drought tolerance and resistance mechanisms that allow better yields in sub optimal conditions, thereby avoiding conservative strategies that sensitively close the stomata to maintain tissue water status, but limit photosynthesis thereby incurring yield penalties.

Acknowledgments We thank the European Union (Rootopower Project Grant \# 289365) for supporting research in our laboratory into long-distance JA signalling of drought stress. 
Author contribution Carlos de Ollas drafted the manuscript with editorial contributions from Ian Dodd.

Open Access This article is distributed under the terms of the Creative Commons Attribution 4.0 International License (http://crea tivecommons.org/licenses/by/4.0/), which permits unrestricted use, distribution, and reproduction in any medium, provided you give appropriate credit to the original author(s) and the source, provide a link to the Creative Commons license, and indicate if changes were made.

\section{References}

Abe H, Yamaguchi-Shinozaki K, Urao T, Iwasaki T, Hosokawa D, Shinozaki K (1997) Role of Arabidopsis MYC and MYB homologs in drought- and abscisic acid-regulated gene expression. Plant Cell 9:1859-1868

Abe H, Urao T, Ito T, Seki M, Shinozaki K, Yamaguchi-Shinozaki K (2003) Arabidopsis AtMYC2 (bHLH) and AtMYB2 (MYB) function as transcriptional activators in abscisic acid signaling. Plant Cell 15:63-78. doi:10.1105/tpc.006130

Arbona V, Argamasilla R, Gómez-Cadenas A (2010) Common and divergent physiological, hormonal and metabolic responses of Arabidopsis thaliana and Thellungiella halophila to water and salt stress. J Plant Physiol 167:1342-1350. doi:10.1016/j.jplph. 2010.05.012

Aroca R, Vernieri P, Ruiz-Lozano JM (2008) Mycorrhizal and nonmycorrhizal Lactuca sativa plants exhibit contrasting responses to exogenous $\mathrm{ABA}$ during drought stress and recovery. J Exp Bot 59:2029-2041. doi:10.1093/jxb/ern057

Bandurska H, Stroiński A, Kubiś J (2003) The effect of jasmonic acid on the accumulation of $\mathrm{ABA}$, proline and spermidine and its influence on membrane injury under water deficit in two barley genotypes. Acta Physiol Plant 25:279-285. doi:10.1007/s11738-003-0009-0

Blum A (2015) Towards a conceptual ABA ideotype in plant breeding for water limited environments. Funct Plant Biol 42:502-513. doi:10.1071/FP14334

Brossa R, Lopez-Carbonell M, Jubany-Mari T, Alegre L (2011) Interplay between abscisic acid and jasmonic acid and its role in water-oxidative stress in wild-type, ABA-deficient, JA-deficient, and ascorbate-deficient arabidopsis plants. J Plant Growth Regul 30:322-333. doi:10.1007/s00344-011-9194-Z

Cheng M-C, Liao P-M, Kuo W-W, Lin T-P (2013) The Arabidopsis ETHYLENE RESPONSE FACTOR1 regulates abiotic stressresponsive gene expression by binding to different cis-acting elements in response to different stress signals. Plant Physiol 162:1566-1582. doi:10.1104/pp.113.221911

Daszkowska-Golec A, Szarejko I (2013) Open or close the gatestomata action under the control of phytohormones in drought stress conditions. Front Plant Sci. doi:10.3389/fpls.2013.00138

Dave A, Hernández ML, He Z, Andriotis VME, Vaistij FE, Larson TR, Graham IA (2011) 12-Oxo-phytodienoic acid accumulation during seed development represses seed germination in Arabidopsis. Plant Cell 23:583-599. doi:10.1105/tpc.110.081489

De Ollas C, Hernando B, Arbona V, Gómez-Cadenas A (2013) Jasmonic acid transient accumulation is needed for abscisic acid increase in citrus roots under drought stress conditions. Physiol Plant 147:296-306. doi:10.1111/j.1399-3054.2012.01659.x

De Ollas C, Arbona V, Gómez-Cadenas A (2015) Jasmonoyl isoleucine accumulation is needed for abscisic acid build-up in roots of Arabidopsis under water stress conditions. Plant Cell Environ. doi:10.1111/pce.12536

Dodd IC (2013) Abscisic acid and stomatal closure: a hydraulic conductance conundrum? New Phytol 197:6-8. doi:10.1111/nph. 12052
Draye X, Kim Y, Lobet G, Javaux M (2010) Model-assisted integration of physiological and environmental constraints affecting the dynamic and spatial patterns of root water uptake from soils. J Exp Bot 61:2145-2155. doi:10.1093/jxb/erq077

Dubois M, Skirycz A, Claeys H, Maleux K, Dhondt S, De Bodt S, Vanden Bossche R, De Milde L, Yoshizumi T, Matsui M, Inzé D (2013) Ethylene Response Factor6 acts as a central regulator of leaf growth under water-limiting conditions in Arabidopsis. Plant Physiol 162:319-332. doi:10.1104/pp.113.216341

Fujita Y, Fujita M, Shinozaki K, Yamaguchi-Shinozaki K (2011) ABA-mediated transcriptional regulation in response to osmotic stress in plants. J Plant Res 124:509-525. doi:10.1007/s10265011-0412-3

Ghanem ME, Albacete A, Smigocki AC, Frébort I, Pospíilová H, Martínez-Andújar C, Acosta M, Sánchez-Bravo J, Lutts S, Dodd IC, Pérez-Alfocea F (2011) Root-synthesized cytokinins improve shoot growth and fruit yield in salinized tomato (Solanum lycopersicum L.) plants. J Exp Bot 62:125-140. doi:10.1093/jxb/erq266

Harb A, Krishnan A, Ambavaram MMR, Pereira A (2010) Molecular and physiological analysis of drought stress in Arabidopsis reveals early responses leading to acclimation in plant growth. Plant Physiol 154:1254-1271. doi:10.1104/pp.110.161752

Hirayama T, Shinozaki K (2010) Research on plant abiotic stress responses in the post-genome era: past, present and future. Plant J 61:1041-1052. doi:10.1111/j.1365-313X.2010.04124.X

Hossain MA, Munemasa S, Uraji M, Nakamura Y, Mori IC, Murata Y (2011) Involvement of endogenous abscisic acid in methyl jasmonate-induced stomatal closure in Arabidopsis. Plant Physiol 156:430-438. doi:10.1104/pp.111.172254

Hu H, Dai M, Yao J, Xiao B, Li X, Zhang Q, Xiong L (2006) Overexpressing a NAM, ATAF, and CUC (NAC) transcription factor enhances drought resistance and salt tolerance in rice. Proc Natl Acad Sci USA 103:12987-12992. doi:10.1073/pnas. 0604882103

Jeong JS, Kim YS, Baek KH, Jung H, Ha S-H, Do Choi Y, Kim M, Reuzeau C, Kim J-K (2010) Root-specific expression of OsNAC10 improves drought tolerance and grain yield in rice under field drought conditions. Plant Physiol 153:185-197. doi: $10.1104 / \mathrm{pp} .110 .154773$

Jiang F, Hartung W (2008) Long-distance signalling of abscisic acid (ABA): the factors regulating the intensity of the ABA signal. J Exp Bot 59:37-43. doi:10.1093/jxb/erm127

Jiang H, Li H, Bu Q, Li C (2009) The RHA2a-interacting proteins anac019 and anac055 may play a dual role in regulating aba response and jasmonate response. Plant Signal Behav 4:464-466. doi:10.4161/psb.4.5.8543

Kazan K (2015) Diverse roles of jasmonates and ethylene in abiotic stress tolerance. Trends Plant Sci 20:219-229. doi:10.1016/j. tplants.2015.02.001

Kazan K, Manners JM (2013) MYC2: the master in action. Mol Plant 6:686-703. doi:10.1093/mp/sss 128

Kim EH, Kim YS, Park S-H, Koo YJ, Choi YD, Chung Y-Y, Lee I-J, Kim J-K (2009) Methyl jasmonate reduces grain yield by mediating stress signals to alter spikelet development in rice 1[W][OA]. Plant Physiol 149:1751-1760. doi:10.1104/pp.108.134684

Kramer PJ (1988) Measurement of plant water status: historical perspectives and current concerns. Irrig Sci 9:275-287. doi:10. 1007/BF00296703

Kudoyarova G, Veselova S, Hartung W, Farhutdinov R, Veselov D, Sharipova G (2011) Involvement of root ABA and hydraulic conductivity in the control of water relations in wheat plants exposed to increased evaporative demand. Planta 233:87-94. doi:10.1007/s00425-010-1286-7

Liu Q, Zhao N, Yamaguch-Shinozaki K, Shinozaki K (2000) Regulatory role of DREB transcription factors in plant drought, salt and cold tolerance. Chin Sci Bull 45:970-975 
Liu L, Wei J, Zhang M, Zhang L, Li C, Wang Q (2012) Ethylene independent induction of lycopene biosynthesis in tomato fruits by jasmonates. J Exp Bot 63:5751-5762. doi:10.1093/jxb/ers224

Lorenzo O, Piqueras R, Sánchez-Serrano JJ, Solano R (2003) ETHYLENE RESPONSE FACTOR1 integrates signals from ethylene and jasmonate pathways in plant defense. Plant Cell 15:165-178. doi: $10.1105 /$ tpc. 007468

Lorenzo O, Chico JM, Sánchez-Serrano JJ, Solano R (2004) JASMONATE-INSENSITIVE1 encodes a MYC transcription factor essential to discriminate between different jasmonateregulated defense responses in arabidopsis. Plant Cell 16:1938-1950. doi:10.1105/tpc.022319

Moffat CS, Ingle RA, Wathugala DL, Saunders NJ, Knight H, Knight MR (2012) ERF5 and ERF6 play redundant roles as positive regulators of JA/Et-mediated defense against Botrytis cinerea in Arabidopsis. PLoS One 7:e35995. doi:10.1371/journal.pone.0035995

Montillet J-L, Leonhardt N, Mondy S, Tranchimand S, Rumeau D, Boudsocq M, Garcia AV, Douki T, Bigeard J, Laurière C, Chevalier A, Castresana C, Hirt H (2013) An abscisic acidindependent oxylipin pathway controls stomatal closure and immune defense in arabidopsis. PLoS Biol. doi:10.1371/journal. pbio. 1001513

Munemasa S, Oda K, Watanabe-Sugimoto M, Nakamura Y, Shimoishi Y, Murata Y (2007) The coronatine-insensitive 1 mutation reveals the hormonal signaling interaction between abscisic acid and methyl jasmonate in Arabidopsis guard cells. Specific impairment of ion channel activation and second messenger production. Plant Physiol 143:1398-1407. doi:10. 1104/pp.106.091298

Munemasa S, Mori IC, Murata Y (2011) Methyl jasmonate signaling and signal crosstalk between methyl jasmonate and abscisic acid in guard cells. Plant Signal Behav 6:939-941

Munemasa S, Hauser F, Park J, Waadt R, Brandt B, Schroeder JI (2015) Mechanisms of abscisic acid-mediated control of stomatal aperture. Curr Opin Plant Biol 28:154-162. doi:10.1016/j. pbi.2015.10.010

Nakashima K, Tran L-SP, Van Nguyen D, Fujita M, Maruyama K, Todaka D, Ito $\mathrm{Y}$, Hayashi N, Shinozaki K, YamaguchiShinozaki K (2007) Functional analysis of a NAC-type transcription factor OsNAC6 involved in abiotic and biotic stressresponsive gene expression in rice. Plant J 51:617-630. doi:10. 1111/j.1365-313X.2007.03168.x

Nakashima K, Takasaki H, Mizoi J, Shinozaki K, YamaguchiShinozaki K (2012) NAC transcription factors in plant abiotic stress responses. Biochim Biophys Acta 1819:97-103. doi:10. 1016/j.bbagrm.2011.10.005

Nambara E, Marion-Poll A (2005) Abscisic acid biosynthesis and catabolism. Annu Rev Plant Biol 56:165-185. doi:10.1146/ annurev.arplant.56.032604.144046

Ohnishi T, Sugahara S, Yamada T, Kikuchi K, Yoshiba Y, Hirano H-Y, Tsutsumi N (2005) OsNAC6, a member of the NAC gene family, is induced by various stresses in rice. Genes Genet Syst 80:135-139. doi:10.1266/ggs.80.135

Pantin F, Monnet F, Jannaud D, Costa JM, Renaud J, Muller B, Simonneau T, Genty B (2013) The dual effect of abscisic acid on stomata. New Phytol 197:65-72. doi:10.1111/nph.12013

Passioura JB (1996) Drought and drought tolerance. Plant Growth Regul 20:79-83

Puértolas J, Alcobendas R, Alarcón JJ, Dodd IC (2013) Long-distance abscisic acid signalling under different vertical soil moisture gradients depends on bulk root water potential and average soil water content in the root zone. Plant Cell Environ 36:1465-1475. doi:10.1111/pce.12076

Puranik S, Sahu PP, Srivastava PS, Prasad M (2012) NAC proteins: regulation and role in stress tolerance. Trends Plant Sci 17:369-381. doi:10.1016/j.tplants.2012.02.004
Rabara RC, Tripathi P, Reese RN, Rushton DL, Alexander D, Timko MP, Shen QJ, Rushton PJ (2015) Tobacco drought stress responses reveal new targets for Solanaceae crop improvement. BMC Genom 16:484. doi:10.1186/s12864-015-1575-4

Roychoudhury A, Paul S, Basu S (2013) Cross-talk between abscisic acid-dependent and abscisic acid-independent pathways during abiotic stress. Plant Cell Rep 32:985-1006. doi:10.1007/s00299013-1414-5

Sánchez-Romera B, Ruiz-Lozano JM, Li G, Luu D-T, MartínezBallesta MDC, Carvajal M, Zamarreño AM, García-Mina JM, Maurel C, Aroca R (2014) Enhancement of root hydraulic conductivity by methyl jasmonate and the role of calcium and abscisic acid in this process. Plant Cell Environ 37:995-1008. doi:10.1111/pce.12214

Savchenko T, Kolla VA, Wang C-Q, Nasafi Z, Hicks DR, Phadungchob B, Chehab WE, Brandizzi F, Froehlich J, Dehesh K (2014) Functional convergence of oxylipin and abscisic acid pathways controls stomatal closure in response to drought. Plant Physiol 164:1151-1160. doi:10.1104/pp.113.234310

Seo J-S, Joo J, Kim M-J, Kim Y-K, Nahm BH, Song SI, Cheong J-J, Lee JS, Kim J-K, Choi YD (2011) OsbHLH148, a basic helixloop-helix protein, interacts with OsJAZ proteins in a jasmonate signaling pathway leading to drought tolerance in rice. Plant $\mathrm{J}$ 65:907-921. doi:10.1111/j.1365-313X.2010.04477.x

Sewelam N, Kazan K, Thomas-Hall SR, Kidd BN, Manners JM, Schenk PM (2013) Ethylene response factor 6 is a regulator of reactive oxygen species signaling in Arabidopsis. PLoS One 8:e70289. doi:10.1371/journal.pone.0070289

Shatil-Cohen A, Attia Z, Moshelion M (2011) Bundle-sheath cell regulation of xylem-mesophyll water transport via aquaporins under drought stress: a target of xylem-borne ABA? Plant J 67:72-80. doi:10.1111/j.1365-313X.2011.04576.X

Suhita D, Raghavendra AS, Kwak JM, Vavasseur A (2004) Cytoplasmic alkalization precedes reactive oxygen species production during methyl jasmonate- and abscisic acid-induced stomatal closure. Plant Physiol 134:1536-1545. doi:10.1104/pp. 103.032250

Tardieu F (2012) Plant tolerance to drought, the solutions exist in nature [Tolérance des plantes à la sécheresse, des solutions existent dans la nature]. Biofutur 42-44 + 46

Thompson AJ, Jackson AC, Symonds RC, Mulholland BJ, Dadswell AR, Blake PS, Burbidge A, Taylor IB (2000) Ectopic expression of a tomato 9-cis-epoxycarotenoid dioxygenase gene causes over-production of abscisic acid. Plant J 23:363-374. doi:10. 1046/j.1365-313X.2000.00789.x

Thompson AJ, Mulholland BJ, Jackson AC, McKee JMT, Hilton HW, Symonds RC, Sonneveld T, Burbidge A, Stevenson P, Taylor IB (2007) Regulation and manipulation of ABA biosynthesis in roots. Plant Cell Environ 30:67-78. doi:10.1111/j.1365-3040. 2006.01606.x

Umezawa T, Nakashima K, Miyakawa T, Kuromori T, Tanokura M, Shinozaki K, Yamaguchi-Shinozaki K (2010) Molecular basis of the core regulatory network in ABA responses: sensing, signaling and transport. Plant Cell Physiol 51:1821-1839. doi:10.1093/pcp/pcq156

Vadez V (2014) Root hydraulics: the forgotten side of roots in drought adaptation. Field Crop Res 165:15-24. doi:10.1016/j.fcr. 2014.03.017

Wasternack C (2014) Action of jasmonates in plant stress responses and development-applied aspects. Biotechnol Adv 32:31-39. doi:10.1016/j.biotechadv.2013.09.009

Weiste C, Iven T, Fischer U, Oñate-Sánchez L, Dröge-Laser W (2007) In planta ORFeome analysis by large-scale over-expression of GATEWAY ${ }^{\circledR}$-compatible cDNA clones: screening of ERF transcription factors involved in abiotic stress defense. Plant J 52:382-390. doi:10.1111/j.1365-313X.2007.03229.x 
Weng J-H, Chien C-T, Chen C-W, Lai X-M (2011) Effects of osmotic- and high-light stresses on PSII efficiency of attached and detached leaves of three tree species adapted to different water regimes. Photosynthetica 49:555-563. doi:10.1007/ s11099-011-0072-5

Wilkinson S, Davies WJ (2002) ABA-based chemical signalling: the co-ordination of responses to stress in plants. Plant Cell Environ 25:195-210. doi:10.1046/j.0016-8025.2001.00824.x

Yamaguchi-Shinozaki K, Shinozaki K (2006) Transcriptional regulatory networks in cellular responses and tolerance to dehydration and cold stresses. Annu Rev Plant Biol 57:781-803. doi:10. 1146/annurev.arplant.57.032905.105444

Yang X, Wang X, Ji L, Yi Z, Fu C, Ran J, Hu R, Zhou G (2015) Overexpression of a Miscanthus lutarioriparius NAC gene MINAC5 confers enhanced drought and cold tolerance in Arabidopsis. Plant Cell Rep 34:943-958. doi:10.1007/s00299015-1756-2
Yoshida T, Mogami J, Yamaguchi-Shinozaki K (2014) ABAdependent and $\mathrm{ABA}$-independent signaling in response to osmotic stress in plants. Curr Opin Plant Biol 21:133-139. doi:10.1016/j.pbi.2014.07.009

Zhang W, Ruan J, Ho T-HD, You Y, Yu T, Quatrano RS (2005) Cisregulatory element based targeted gene finding: genome-wide identification of abscisic acid- and abiotic stress-responsive genes in Arabidopsis thaliana. Bioinformatics 21:3074-3081. doi:10.1093/bioinformatics/bti490

Zhang L, Gao M, Hu J, Zhang X, Wang K, Ashraf M (2012) Modulation role of abscisic acid (ABA) on growth, water relations and glycinebetaine metabolism in two maize (zea mays L.) cultivars under drought stress. Int J Mol Sci 13:3189-3202. doi:10.3390/ijms 13033189 